\title{
PARTIALLY ORDERED TOPOLOGICAL SPACES
}

\author{
L. E. WARD, JR. ${ }^{1}$
}

1. Introduction. In this paper we shall consider topological spaces endowed with a reflexive, transitive, binary relation, which, following Birkhoff $[1],^{2}$ we shall call a quasi order. It will frequently be assumed that this quasi order is continuous in an appropriate sense (see $\S 2)$ as well as being a partial order. Various aspects of spaces possessing a quasi order have been investigated by L. Nachbin $[6 ; 7 ; 8]$, Birkhoff [1, pp. 38-41, 59-64, 80-82, as well as the papers cited on those pages], and the author [13]. In addition, Wallace [10] has employed an argument using quasi ordered spaces to obtain a fixed point theorem for locally connected continua. This paper is divided into three sections, the first of which is concerned with definitions and preliminary results. A fundamental result due to Wallace, which will have later applications, is proved. $\S 3$ is concerned with compactness and connectedness properties of chains contained in quasi ordered spaces, and $\$ 4$ deals with fixed point theorems for quasi ordered spaces. As an application, a new theorem on fixed sets for locally connected continua is proved. This proof leans on a method first employed by Wallace [10].

The author wishes to acknowledge his debt and gratitude to Professor A. D. Wallace for his patient and encouraging suggestions during the preparation of this paper.

2. Definitions and preliminary results. By a quasi order on a set $X$, we mean a reflexive, transitive binary relation, $\leqq$. If this relation is also anti-symmetric, it is a partial order. If a quasi order satisfies the following linearity law

$$
\text { if } x, y \in X \text {, then } x \leqq y \text { or } y \leqq x,
$$

then it is said to be a linear quasi order. We write $x<y$ when $x \leqq y$ and $x \neq y$. Henceforth, the following notation will be standard: If $X$ is a quasi ordered set, and $A$ is a subset of $X$, we write

$$
\begin{gathered}
L(A)=\{y \in X: y \leqq x \text { for some } x \in A\}, \\
M(A)=\{y \in X: x \leqq y \text { for some } x \in A\}, \\
\\
E(A)=L(A) \cap M(A) .
\end{gathered}
$$

Presented to the Society, December 28, 1952; received by the editors June 8, 1953.

1 This work was done under Contract N7-onr-434, Task Order No. III, Navy Department (Office of Naval Research).

2 Numbers in brackets refer to bibliography. 
$L(A)$ is called the set of predecessors of $A$, and $M(A)$ the set of successors of $A$. Clearly, $A \subset E(A)$; if $A=L(A)(M(A))$, we say that $A$ is monotone decreasing (increasing) or simply decreasing (increasing). We note that if $A$ is decreasing (increasing), then $X-A$ is increasing (decreasing). Also, the union or intersection of a family of increasing (decreasing) sets is increasing (decreasing). Obviously, $L(A)(M(A))$ is decreasing (increasing).

Suppose now that $X$ is a topological space endowed with a quasi order. The quasi order is lower (upper) semicontinuous provided, whenever $a b(b a)$ in $X$, there is an open set $U$, with $a \in U$, such that if $x \in U$ then $x \quad b$ ( $b x$ ). The quasi order is semicontinuous if it is both upper and lower semicontinuous. It is continuous provided, whenever $a \ddagger b$ in $X$, there are open sets $U$ and $V, a \in U$ and $b \in V$, such that if $x \in U$ and $y \in V$, then $x$. A quasi ordered topological space (hereafter abbreviated QOTS) is a topological space together with a semicontinuous quasi order. If the quasi order is a partial order, then the space is called a partially ordered topological space (hereafter abbreviated POTS). Clearly, the statement that $X$ is a QOTS is equivalent to the assertion that $L(x)$ and $M(x)$ are closed sets, for each $x \in X$.

Lemma 1. If $X$ is a topological space with a quasi order, then the following statements are equivalent:

(1) the quasi order is continuous,

(2) the graph of the quasi order is a closed set in $X \times X$,

(3) if $a \$ b$ in $X$, then there are neighborhoods $N$ and $N^{\prime}$ of $a$ and $b$ respectively, such that $N$ is increasing, $N^{\prime}$ is decreasing, and $N \cap N^{\prime}=0$.

Lemma 2. A continuous quasi order is semicontinuous. A POTS is a $T_{1}$-space; a POTS with continuous partial order is a Hausdorff space.

The proofs of Lemmas 1 and 2 are trivial. We note also

Lemma 3. If $X$ is a topological space with a linear quasi order, then continuity and semicontinuity of the quasi order are equivalent.

Proof. By Lemma 2 it suffices to show that semicontinuity implies continuity. If $a \vdots b$, then $b \leqq a$ and $b \notin E(a)$. We shall exhibit open sets $U$ and $V, a \in U, b \in V$, such that if $x \in U$ and $y \in V$, then $x \leqq y$. If there exists $c \in X$ such that $b \leqq c \leqq a, c \notin E(a) \cup E(b)$, let $U=X-L(c), \quad V=X-M(c)$. Otherwise, let $U=X-L(b), \quad V=X$ $-M(a)$. It is clear that $U$ and $V$ have the desired properties.

We define a chain to be a subset of a quasi ordered set which is linear with respect to the quasi order. A maximal chain is a chain which is properly contained in no other chain. The Hausdorff maxi- 
mality principle ( $=$ Zorn's lemma) assures the existence of maximal chains in any quasi ordered set. A useful result of Wallace [10] is the following

LEMma 4. Every maximal chain in a QOTS is a closed set.

Proof. Let $C$ be a maximal chain in a QOTS. Then

$$
C=\cap\{L(x) \cup M(x): x \in C\} .
$$

By semicontinuity, $C$ is closed.

An element $y$ in a quasi ordered set $X$ is minimal (maximal), whenever $x \leqq y(y \leqq x)$ in $X$ implies $y \leqq x(x \leqq y)$. The following fundamental theorem was first proved in [10].

THEOREM 1. A non-null compact space endowed with a lower (upper) semicontinuous quasi order has a minimal (maximal) element.

Proof. We give the proof for the lower semicontinuous case. Let

$$
\mathfrak{l}=\{L(x): x \in X\},
$$

where $X$ is a non-null compact space with lower semicontinuous quasi order. $\&$ being partially ordered by the inclusion relation, there exists $\mathfrak{M C R}$, where $\mathfrak{M}$ is a maximal chain with respect to the inclusion relation. By lower semicontinuity, each $L(x)$ is closed, and therefore, since $X$ is compact, there exists

$$
x_{0} \in \cap\{L(x): L(x) \in M\} .
$$

By the maximality of $\mathfrak{M}, x_{0}$ is minimal.

This theorem is asserted by Birkhoff in the partially ordered case in a somewhat different (but equivalent) form [1, Chap. IV, Theorem 16]. The theorem has an interesting application in the following corollary, due originally to R. L. Moore. The proof given below, with modifications, follows that of [15, Chap. I, 12.15]. A continuum is a compact connected Hausdorff space.

Corollary 1.1. A nondegenerate continuum has at least two noncutpoints.

Proof. Let $X$ be a nondegenerate continuum, and $N$ its set of non-cutpoints. If $N$ contains at most one point, then there exists $x_{0} \in X-N$; hence $X-x_{0}=A \cup B, A \mid B,{ }^{3} A$ and $B$ non-null. We may assume $N \subset B$; then for each $x \in A$, there is a decomposition

\footnotetext{
3 $A \mid B$ if, and only if, $A$ and $B$ are separated sets, i.e., $\bar{A} \cap B=0=A \cap \bar{B}$.
} 


$$
X-x=A(x) \cup B(x), \quad A(x) \mid B(x),
$$

with $x_{0} \in B(x)$, so that $A(x) \subset A$. Define a partial order in $\bar{A}$ by $x \leqq y$ if, and only if $A(x) \subset A(y)$. Since $L(x)=\bar{A}(x)$, this partial order is lower semicontinuous. (As a matter of fact, the partial order is semicontinuous, but this is not essential to the proof.) $\bar{A}$ being compact, there is a minimal element $p \in \bar{A}$. Since $p$ is minimal, $A(p)=0$, a contradiction.

A subset $A$ of a QOTS, $X$, is convex provided $A=E(A) . X$ is quasilocally convex (abbreviated $q l c$ ) provided, whenever $x \in X$ and $E(x)$ $\subset U$, an open set, there is a convex open set $V$ such that $E(x) \subset V \subset U$. $X$ is locally convex provided, whenever $x \in X$ and $x \in U$, an open set, there is a convex open set $V$ such that $x \in V \subset U$. If $Y$ is a quasi ordered set, a function $f: X \rightarrow Y$ is order-preserving provided $f(a) \leqq f(b)$ in $Y$ whenever $a \leqq b$ in $X$.

Nachbin $[6 ; 7 ; 8]$ has shown that if $X$ is a compact POTS with continuous partial order, and $b \leq a$ in $X$, then there is a continuous order-preserving $f: X \rightarrow I$ (the unit interval) such that $f(a)=0, f(b)=1$. The author [13] observed that this result can easily be extended to the case where $X$ is a compact QOTS with continuous quasi order. It follows that

TheOREM 2. A compact Hausdorff QOTS with continuous quasi order is qlc.

Proof. Let $X$ be a compact Hausdorff QOTS with continuous quasi order, $x \in X, E(x) \subset U$, an open set. If $t \in X-U$, then either $t \$ x$ or $x \neq t$. If $t \leq x$, then there is a continuous order-preserving $f_{t}: X \rightarrow I$ such that $f_{t}(x)=0, f_{t}(t)=1$. Let

$$
U_{t}=\left\{y: f_{t}(y)<1 / 2\right\}
$$

then $x \in U_{t}$, a decreasing open set, and $t \in \bar{U}_{t}$. In a similar fashion, if $x \neq t$, then we obtain $U_{t}$, an open increasing set. Since $X-U$ is compact, there is a finite set $t_{1}, t_{2}, \cdots, t_{n} \in X-U$ such that

$$
X-U \subset \bigcup_{i=1}^{n}\left\{X-\bar{U}_{t_{i}}\right\}
$$

Letting

$$
V=\bigcap_{i=1}^{n}\left\{U_{t_{i}}\right\}
$$

we see that $V$ is convex and open and $E(x) \subset C V \subset U$. 
Corollary 2.1 (NachBin). A compact POTS with continuous partial order is locally convex.

3. Chains in quasi ordered spaces. In subsequent theorems, it will frequently be the case that compactness is an unnecessarily strong hypothesis; rather, compactness of maximal chains will suffice. In Theorem 3, below, the meaning of this condition is analyzed. The subject of chains satisfying the anti-symmetry condition (i.e., $E(x)$ $=x$ for every element $x$ ) has been thoroughly studied for the case of the interval topology, that is, the topology which has for a base for the open sets all the open intervals $(a, b)$ where

$$
(a, b)=\{x: a<x<b\} .
$$

This topology is the coarsest topology for which the order is continuous. The study of chains arose as a generalization of the real numbers and can, within limitations, be extended to quasi ordered spaces. (On the study of chains in connection with this paper, see $[1$, Chap. III; $4 ; 2]$. Also, relative to lattices with the interval topology, see [3].)

Let $A$ be a subset of a quasi ordered set $X$. The element $x \in X$ is an upper (lower) bound for $A$ provided $a \leqq x(x \leqq a)$ for all $a \in A$. The element $x$ is a least upper (greatest lower) bound for $A$ if $x$ is a minimal (maximal) element of the set of upper (lower) bounds of $A$. Hereafter, we abbreviate lub. and glb. for least upper and greatest lower bound. It was shown in [4] and [1] that an anti-symmetric chain is complete if, and only if, it is compact in its interval topology. Subsequently, Frink [3] generalized this theorem by showing that, in a lattice, compactness in the interval topology is equivalent to completeness in the customary lattice-theoretic sense. These theorems fail for topologies finer than the interval topology.

It is possible to define an "interval" topology for quasi ordered sets. It is that topology which has for a subbase for closed sets all sets of the form $L(x)$ or $M(x)$, where $x$ is a member of the quasi ordered set. Again this interval topology is the coarsest topology for which the quasi order is semicontinuous. We say that a quasi ordered set is complete if every chain of the set possesses a lub. and a glb. For any chain $C$ of a quasi ordered set, we denote by $\max C$ the set of maximal elements of $C$, and by $\min C$ the set of minimal elements of $C$. If $A$ and $B$ are subsets of a quasi ordered set, we write $A<B$ when, for each $a \in A, b \in B$, we have $a \leqq b$ and $a \notin E(b)$.

We now extend the result of Haar and König [4] to the case of quasi ordered spaces, at the same time gaining some insight into the compact maximal chain condition. 
ThEOREM 3. Let $X$ be a QOTS. Then the following statements are equivalent:

(1) if $C$ is a closed chain in $X$, then $C$ is compact,

(2) if $C$ is a maximal chain in $X$, then $C$ is compact,

(3) if $C$ is a non-null closed chain in $X$, then $\max C$ and $\min C$ are non-null compact sets,

(4) $E(x)$ is compact for each $x \in X$, and every closed subset of $X$ has maximal and minimal elements.

The statements (1) to (4) imply

(5) $X$ is complete and $E(x)$ is compact for each $x \in X$.

If $X$ is a chain with interval topology, then (5) implies the statements (1) to (4).

Proof. In view of Lemma 4 , it is obvious that (1) and (2) are equivalent. By Theorem 1 and the semicontinuity of the quasi order, (1) implies (3).

(3) implies (1): Let $C$ be a non-null closed chain in $X, \mathfrak{T}$ a family of non-null closed subsets of $C$, linearly ordered by inclusion. Let

$$
\Im=\{E(T) \cap C: T \in \mathfrak{T}\} .
$$

We assert that each member of $\subseteq$ is closed; for suppose $x \in C-E(T)$ for some $T \in \mathfrak{T}$. By (3), $\min T \neq 0 \neq \max T$, and hence either $x$ $\prec \min T$, or $\max T \prec x$, or $\min T \prec x \prec \max T$. If $x<\min T$, then $x \in C-M(\min T)$; now $M(\min T)$ is a closed set containing $E(T)$, so that $x \in C-\bar{E}(T)$. A similar argument follows if $\max T<x$. If $\min T<x<\max T$, let $T_{0}=L(x) \cap T, T_{1}=M(x) \cap T$. Then $T_{0}$ and $T_{1}$ are disjoint closed sets whose union is $T, \max T_{0}<x$, and $x<\min T_{1}$. Following the argument above,

$$
x \in C-\bar{E}\left(T_{0}\right) \cup \bar{E}\left(T_{1}\right)=C-\bar{E}(T) .
$$

Hence $E(T) \cap C=\bar{E}(T) \cap C$, so that each member of $\subseteq$ is closed. Let

$$
M=U\{\min S: S \in \Im\}, \quad U=\bigcup\{\max S: S \in \Im\} .
$$

By (3), we may choose $m_{0} \in \max \bar{M}$. By semicontinuity, $m_{0} \leqq u$, for each $u \in U$. Suppose there exists $S_{1} \in \mathbb{S}$ such that $m_{0} \notin S_{1}$; then $\min S_{1} \prec m_{0}<\max S_{1}$. Denoting $K=S_{1} \cap L\left(m_{0}\right)$, it is clear that $0 \neq \max K<m_{0}$. For $S \in \subseteq$, either $S_{1} \subset S$ or $S \subset S_{1}$. If $S_{1} \subset S$, then $\max K \subset S_{1} \subset S$, so that $\min S \subset L(K)$. If $S \subset S_{1}$, then min $S \subset S_{1}$ $\cap M \subset K$. In either event, $\min S \subset L(K)$, so that $M \subset L(K)$. Now $L(K)$ is a closed set and $m_{0} \in L(K)$, contradicting $m_{0} \in \bar{M}$. Evidently $m_{0} \in S_{1}$ for each $S_{1} \in \mathfrak{S}$. Clearly, then,

$$
\left\{E\left(m_{0}\right) \cap T: T \in \mathfrak{T}\right\}
$$


is a family of non-null closed sets, linearly ordered by inclusion. By (3), $E\left(m_{0}\right)$ is compact, so that

$$
\cap\left\{E\left(m_{0}\right) \cap T: T \in \mathfrak{T}\right\} \neq 0 .
$$

Hence $\mathfrak{T}$ has non-null intersection, so that $C$ is compact.

The equivalence of (3) and (4) follows by routine arguments. To show (1) implies (5), we note that since each $E(x)$ is a chain, each $E(x)$ is compact by (1). Let $C$ be a chain of $X$; then $\bar{C}$ is a compact chain, and hence by Theorem $1, \bar{C}$ contains maximal and minimal elements, say $x_{1}$ and $x_{0}$. It is obvious that $x_{1}$ is an upper bound for $C$, and by semicontinuity, $x_{1}$ is a lub. for $C$. Similarly, $x_{0}$ is a glb. for $C$, proving (5).

If $X$ is a chain with interval topology, then (5) implies (3): let $C$ be a closed subchain of $X$. In view of (5) it suffices to show that $\min C$, $\max C$ are non-null. By (5), $C$ has a lub. and a glb., say $x_{1}$ and $x_{0}$. If $x_{1} \in C$, then, since $X$ has the interval topology, there exists $a \in X$ such that $C \subset L(a), x_{1} \in X-L(a)$. But then $a$ is an upper bound for $C$, contradicting that $x_{1}$ is a lub. Similarly, $x_{0} \in C$. This completes the proof.

It is not true that (5) implies (3), even when $X$ is a partially ordered set with the interval topology. We indicate a counter example. Let $X=B \cup C$ where $C$ is the real interval

$$
C=\{t: 0 \leqq t<1\} \text {, }
$$

with the usual linear order, and $B$ is a set of two elements, $x$ and $y$. Define $x$ and $y$ to be successors of each element of $C$, but not comparable with each other. Then $X$ is complete, $x$ and $y$ are lub.'s for $C$, and $C$ is closed in the interval topology, since $C=L(x) \cap L(y)$. However, $x$ and $y$ are not members of $C$, so that $\max C=0$.

A partially ordered set $X$ is dense in the sense of order, or, more simply, order dense provided, whenever $x<y$ in $X$, there exists $z \in X$ such that $x<z<y$. In the remainder of this section we investigate conditions sufficient to insure the connectedness of POTS's and their maximal chains. Further results of this sort can be found in [13]. A related question has been solved by Eilenberg [2] who showed that a connected Hausdorff space admits a continuous linear order if, and only if, the complement of the diagonal in $X \times X$ is not connected. Analogues of the following results are well known for linear order; the reader is referred to the previously mentioned bibliography of [1].

Theorem 4. A connected chain in a POTS, $X$, is order dense. If $X$ has compact maximal chains, then any order dense maximal chain is connected. 
Proof. If $C$ is a non-order dense chain in $X$, choose $x<y$ in $C$ with $M(x) \cap L(y)=x \cup y$. Then

$$
C \subset L(x) \cup M(y), \quad L(x) \mid M(y)
$$

so that $C$ is not connected. Conversely, if $X$ has compact maximal chains, suppose $C$ is a non-connected maximal chain of $X$. Then $C=P \cup Q, P \mid Q, P \neq 0 \neq Q$. Since $C$ is compact, by Theorem $1, C$ contains a maximal element, $u$. Assuming $u \in Q$, we know by the compactness of $P$ that $P$ contains a maximal element $p<u$. Let $P^{\prime}=L(p) \cap C, Q^{\prime}=C-P^{\prime}$. Readily, $P^{\prime} \mid Q^{\prime}$, and $Q^{\prime}$ contains a minimal element, $q$. By the maximality of $C, M(p) \cap L(q)=p \cup q$, so that $C$ fails to be order dense.

Theorem 5. Let $X$ be an order dense POTS with compact maximal chains, and suppose either the set of maximal elements or the set of minimal elements of $X$ is connected. Then $X$ is connected.

Proof. If $X=P \cup Q, P \mid Q, P \neq 0 \neq Q$, we may assume the maximal elements of $X$ are connected and contained in $Q$. Let $C$ be a maximal chain meeting $P$; then $C$ is not connected, in contradiction of Theorem 4 . Hence $X$ is connected.

TheOREM 6. Let $X$ be a POTS with compact maximal chains. Then a necessary and sufficient condition that every maximal chain be connected is that $L(x) \cap M(y)$ be connected for every pair of elements $x$, $y \in X$.

Proof. The necessity of the condition is obvious, and is valid in case $X$ is a QOTS without conditions on the maximal chains. To prove the sufficiency, suppose $X$ contains a non-connected maximal chain, $C$. As in Theorem 4, we obtain $p, q \in C$ such that $M(p) \cap L(q)=p \cup q$, contradicting the condition that $M(p) \cap L(q)$ be connected.

It might reasonably be conjectured that if $X$ is a compact POTS in which $M(x)$ and $L(x)$ are connected for every $x \in X$, then every element of $X$ is an element of some connected maximal chain. That this is not true is demonstrated by the following example. Let $X$ $=I \times I$, the Cartesian product of the unit interval with itself, with the usual plane topology. Define $(a, b) \leqq(c, d)$ if, and only if, one of the following three conditions is satisfied:

$$
\begin{array}{ll}
a=c, & b \leqq d, \\
a \leqq c, & b=0, \\
a=0, & d=1 .
\end{array}
$$

It is simple (but tedious) to verify that the relation so defined is 
a semicontinuous partial order. We note in addition that $(0,0)$ is the only minimal element of $X$, and that every point $(x, 1)$ with $x>0$ is maximal. Further $L(x, y)$ and $M(x, y)$ are connected for each $(x, y)$ $\in X$. However the only maximal chains containing $(0,1)$ are of the form

$$
(\{0\} \times I) \cup(x, 1), \quad x>0,
$$

and hence are not connected.

4. Remarks on fixed points. In this section we prove a number of theorems on fixed points and sets, and give two applications to obtain theorems on fixed points and sets in locally connected continua. The main result, Theorem 9 , is an improvement of a result due to Schweigert [9] and Wallace [10]. The results on fixed points and sets in QOTS's are similar to a theorem of Wallace [10].

By a net, we mean a function $x: \Lambda \rightarrow X$, where $\Lambda$ is a directed set and $X$ is a set. For $\lambda \in \Lambda$, we write $x_{\lambda}$ to denote the image of $\lambda$ under the function $x$. (For further details on nets, see [5].) If $X$ is a quasi ordered set, we shall denote both the quasi order in $X$ and the direction in $\Lambda$ by the symbol $\leqq$. A net $x$ is monotone increasing (decreasing) if, whenever $\lambda \leqq \mu$ in $\Lambda$, we have $x_{\lambda} \leqq x_{\mu}\left(x_{\mu} \leqq x_{\lambda}\right)$ in $X$. If $X$ is a topological space and $x: \Lambda \rightarrow X$ is a net, we say that $x$ clusters at the point $x_{0} \in X$ provided, whenever $x_{0} \in U$, an open set, and $\lambda \in \Lambda$, there is $\mu \in \Lambda, \lambda \leqq \mu$, such that $x_{\mu} \in U$. The net $x$ converges to $x_{0}$ provided, whenever $x_{0} \in U$, an open set, there is $\lambda \in \Lambda$ such that $x_{\mu} \in U$ for all $\mu \geqq \lambda$.

Lemma 5. Let $X$ be a compact Hausdorff QOTS with continuous quasi order. Then every monotone net in $X$ clusters, and the set of cluster points is contained in $E\left(x_{0}\right)$, for some $x_{0} \in X$.

Proof. Let $x$ be a monotone increasing net in $X$. (The proof for the decreasing case is entirely dual.) Since $X$ is compact, $x$ clusters, say at $x_{0}$. Let $U$ be an open set containing $E\left(x_{0}\right)$; by Theorem $2, X$ is $q l c$, so that there is a convex open set $V$ such that $E\left(x_{0}\right) \subset V \subset U$. Since $x$ clusters at $V$, there is $\lambda_{0} \in \Lambda$ such that $x_{\lambda_{0}} \in V$, and, if $\lambda \geqq \lambda_{0}$, then there is $\lambda^{\prime} \geqq \lambda$ such that $x_{\lambda^{\prime}} \in V$. Since $V$ is convex, $x_{\lambda} \in V \subset U$, for all $\lambda \geqq \lambda_{0}$. Evidently, $x$ can cluster only at points of $E\left(x_{0}\right)$.

Corollary. If $X$ is a compact POTS with continuous order, then every monotone net in $X$ converges.

The proofs of the next two lemmas are trivial.

Lemma 6. Let $X$ be a topological space, $f: X \rightarrow X$ continuous, and $x \in X$ 
such that the sequence $f^{n}(x), n=1,2, \cdots$, clusters at some $x_{0} \in X$. Then $f^{n}(x)$ clusters at $f\left(x_{0}\right)$.

Lemma 7. Let $X$ be a topological space, $f: X \rightarrow X$ continuous, and $x_{n}, n=1,2, \cdots$, a sequence in $X$ such that $x_{n}=f\left(x_{n+1}\right)$. If $x_{n}$ clusters at $x_{0}$, then $x_{n}$ clusters at $f\left(x_{0}\right)$.

Two elements, $x$ and $y$, in a quasi ordered set are comparable, if either $x \leqq y$ or $y \leqq x$. The following result is related to a theorem of Birkhoff [1, Chap. IV, Theorem 8]; see also the references cited in connection with Birkhoff's theorem, in particular, the result of B. Knaster, that an order-preserving function on a complete antisymmetric chain into itself has a fixed point.

THEOREM 7. Let $X$ be a Hausdorff QOTS with compact maximal chains, $f: X \rightarrow X$ continuous and order-preserving. $A$ necessary and sufficient condition that there exist a non-null compact set $K \subset E\left(x_{0}\right)$, for some $x_{0} \in X$, such that $f(K)=K$ is that there exist $x \in X$ such that $x$ and $f(x)$ are comparable.

Proof. The necessity of the condition is obvious. To prove the sufficiency, suppose $x$ exists such that $x$ and $f(x)$ are comparable. Then, since $f$ is order-preserving, the set

$$
\left\{f^{n}(x): n=1,2, \cdots\right\}
$$

is a chain and therefore is contained in a maximal (and hence compact) chain. By Lemma $5, f^{n}(x)$ clusters at some $x_{0}$, and all cluster points are contained in $E\left(x_{0}\right)$. By Lemma 6, $f\left(E\left(x_{0}\right)\right) \subset E\left(x_{0}\right)$. Denote

$$
K=\cap\left\{f^{n}\left(E\left(x_{0}\right)\right): n=1,2, \cdots\right\} .
$$

Clearly $K$ is a non-null compact subset of $E\left(x_{0}\right)$, and $f(K)=K$.

CoROllary 7.1. If $X$ is partially ordered, then a necessary and sufficient condition that $f$ have a fixed point is that there exist $x \in X$ such that $x$ and $f(x)$ are comparable.

If $X$ is a quasi ordered set with an element $e \in X$ such that $e \leqq x$, for all $x \in X$, and $A$ is a subset of $X$, we say that $A$ is bounded away from $e$ provided there is $y \in X-E(e)$ such that $A \subset M(y)$.

Theorem 8. Let $X$ be a Hausdorff QOTS with compact maximal chains, and suppose there exists $e \in X$ such that $e \leqq x$ for all $x \in X$. Let $f: X \rightarrow X$ be continuous, order-preserving, and satisfying

(i) there exists $x \in X-E(e)$ such that $x$ and $f(x)$ are comparable,

(ii) if $x$ satisfies (i), then either the sequence $f^{n}(x), n=1,2, \cdots$, is 
bounded away from $e$, or there exists $y \in X$ such that $x \in E(f(y))$ and $f(y) \leqq y$.

Then there is an $x_{0} \in X-E(e)$ and a non-null compact set $K \subset E\left(x_{0}\right)$ such that $f(K)=K$.

Proof. Choose $x$ satisfying (i). If $f^{n}(x)$ is bounded away from $e$, we obtain $K$ as in Theorem 7 . Otherwise, by (ii), there is a $y_{1} \in X$ such that $x \in E\left(f\left(y_{1}\right)\right), f\left(y_{1}\right) \leqq y_{1}$. Inductively, we obtain a sequence $y_{n}$ such that

$$
x \leqq f\left(y_{1}\right) \leqq y_{1} \leqq f\left(y_{2}\right) \leqq y_{2} \leqq \cdots,
$$

where each $y_{n} \in E\left(f\left(y_{n+1}\right)\right)$. Since $\left\{y_{n}: n=1,2, \cdots\right\}$ is a chain, it is contained in a maximal chain. Therefore, by Lemma 5, $y_{n}$ clusters, and all cluster points are contained in some $E\left(x_{0}\right), x_{0} \in X-E(e)$. By Lemma $7, f\left(E\left(x_{0}\right)\right) \subset E\left(x_{0}\right)$. Defining $K$ as in Theorem 7, the theorem is proved.

Corollary 8.1. If $X$ is partially ordered, then $f$ has a fixed point distinct from $e$.

The condition (ii), or something very like it, cannot be omitted from the hypotheses of Theorem 8 , even if $X$ is compact and $f$ is onto. For let $X=A \cup B$ be the plane set defined by

$$
A=\{(\rho, \theta): \rho=1\}, \quad B=\left\{(\rho, \theta): \rho=\frac{\theta}{\theta+1}\right\} .
$$

Let $e=(0,0)$, and fix $x_{0}=\left(\rho_{0}, \theta_{0}\right) \in B-e$. Partially order $X$ by $\left(\rho_{1}, \theta_{1}\right)$ $\leqq\left(\rho_{2}, \theta_{2}\right)$ if, and only if, $\rho_{1}=\rho_{2}, \theta_{1}=\theta_{2}$, or $\rho_{1}=0$, or $\rho_{1} \leqq \rho_{2} \leqq \rho_{0}$. Thus $X$ is a compact POTS. Define $f: X \rightarrow X$ by

$$
\begin{gathered}
f(1, \theta)=(1, \theta-\pi / 2), \\
f\left(\frac{\theta}{\theta+1}, \theta\right)=\left(\max \left\{0, \frac{\theta-\pi / 2}{\theta+1-\pi / 2}\right\}, \max \{0, \theta-\pi / 2\}\right) .
\end{gathered}
$$

Then $f$ is an order-preserving continuous function onto, and $x_{0}$ and $f\left(x_{0}\right)$ are comparable. However $e$ is the only fixed point under $f$.

Four more results follow easily from Theorems 7 and 8 .

Corollary 8.2. Let $X, f$ be as in Theorem 7 , and suppose $X$ satisfies the equivalent conditions

(i) there exists $u \in X$ such that $L(u)=X$,

(ii) for $x \in X, y \in X$, there exists $z \in X$ such that $x \leqq z$ and $y \leqq z$.

Then there is a non-null compact set $K$ contained in $E\left(x_{0}\right)$, for some $x_{0} \in X$, such that $f(K)=K$. 
Corollary 8.3. If $X, f$ are as in Corollary 8.2, and $X$ is partially ordered, then $f$ has a fixed point.

Corollary 8.4. Let $X$ be a compact Hausdorff QOTS satisfying (i) and (ii) of Corollary 8.2, as well as

(iii) there exists $e \in X$ such that $e \leqq x$ for all $x \in X$, and $E(e) \neq X$.

Then, if $f: X \rightarrow X$ is continuous, order-preserving, and onto, there is a non-null compact set $K \subset E\left(x_{0}\right)$, for some $x_{0} \in X-E(e)$, such that $f(K)=K$.

Corollary 8.5. If $X, f$ are as in Corollary 8.4, and $X$ is partially ordered, then $f$ has a fixed point distinct from $e$.

A point $e$ of a topological space is an endpoint if, whenever $e \in U$, an open set, there is an open set $V$ such that $e \in V \subset \bar{V} \subset U$, and $\bar{V}-V$ is a single point. Two subsets $P$ and $Q$ of a connected space $X$ are separated by the set $K \subset X$ if

$$
X-K=A \cup B, \quad A \mid B, \quad P \subset A, Q \subset B .
$$

If points $p$ and $q$ are not separated by any point, we write $p \sim q$. A prime chain is a continuum which is either an endpoint, a cutpoint, or a nondegenerate set $E$ containing distinct elements $a$ and $b$ with $a \sim b$, and representable as

$$
E=\{x: a \sim x \text { and } x \sim b\} .
$$

An endelement is a prime chain $E$ with the property that if $E \subset U$, an open set, then there is an open set $V$ such that $E \subset \bar{V} \subset U$, and $\bar{V}$ $-V$ is a single point.

LeMma 8. Let $X$ be a connected, locally connected Hausdorff space. If $E$ is an endelement of $X$, then $E$ contains at most one cutpoint of $X$.

Proof. Suppose $E$ contains two distinct cutpoints, $x_{0}$ and $x_{1}$, of $X$. Then for $i=0,1$, we have

$$
X-x_{i}=A_{i} \cup B_{i}, \quad A_{i} \mid B_{i}, \quad E-x_{i} \subset A_{i},
$$

and, by the local connectedness of $X$, we may take $A_{i}$ connected. Clearly, $B_{i}-B_{j} \neq 0$, for $i \neq j$. Choose $y_{i} \in B_{i}-B_{j}(i=0,1, i \neq j)$, and let $C_{i}$ be the component of $X-x_{i}$ such that $y_{i} \in C_{i}$. If $C_{0} \cap C_{1} \neq 0$, then $C_{0} \cup C_{1}$ is connected; since $x_{1} \notin C_{0}$, evidently $C_{0} \subset B_{1}$, contradicting $y_{0} \in B_{0}-B_{1}$. Therefore, $C_{0} \cap C_{1}=0$.

Choose $U$ an open set such that $E \subset U$ and $U$ meets both $C_{0}$ and $C_{1}$ but contains neither. Since $E$ is an endelement, there is an open set $V$ such that $E \subset \bar{V} \subset U$, and $\bar{V}-V$ is a single point. Therefore, 
either $x_{0} \in V$ or $x_{1} \in V$, and it follows easily that $\bar{V}-V$ meets both $C_{0}$ and $C_{1}$, a contradiction.

Lemma 9. If $X$ is a connected, locally connected Hausdorff space, and $E$ is an endelement of $X$ containing a cutpoint $x$ of $X$, then $E-x$ and $X-E$ are separated sets.

Proof. Since $X$ is locally connected, it is sufficient to show that if $C_{0}$ is the component of $X-x$ which contains $E-x$, then $C_{0}=E-x$. If not, there exists $y \in C_{0}-E$. Let $C$ be any component of $X-x$ distinct from $C_{0}$, and $P$ a connected open set such that $x \in P, y \notin P$, $C-P \neq 0$. Then $\left(C_{0} \cup P\right)-y$ is an open set containing $E$, and, since $E$ is an endelement, there is a connected open set $V$ such that $E \subset \bar{V}$ $C\left(C_{0} \cup P\right)-y$, where $\bar{V}-V=p$, a point. Since $y \notin V$, it follows that $p \in C_{0}$, whence $x \in V$. Therefore $(\bar{V}-V) \cap C \neq 0$, contradicting $p \in C_{0}$.

Now suppose $X$ and $Y$ are Hausdorff spaces and $f(X)=Y$ is continuous. $f$ is non-alternating if, for every decomposition

$$
X-f^{-1}\left(y_{0}\right)=M \cup N, \quad M \mid N,
$$

no set $f^{-1}(y)$ meets both $M$ and $N . f$ is monotone if $f^{-1}(y)$ is connected for every $y \in Y$. Obviously, every montone function is non-alternating. The next two lemmas are due to Wallace [11].

LEMma 10. The following are necessary and sufficient conditions for $f$ to be non-alternating: for each decomposition

$$
X-f^{-1}\left(y_{0}\right)=M \cup N, \quad M \mid N,
$$

(1) $M=f^{-1} f(M)$ and $N=f^{-1} f(N)$,

(2) $f(M) \cap f(N)=0$,

(3) $f^{-1} f(M) \cap f^{-1} f(N)=0$.

LEMma 11. If $f$ is closed and non-alternating, and if $f^{-1}(y)$ separates $P$ and $Q$ in $X$, then $y$ separates $f(P)$ and $f(Q)$ in $Y$.

For the next three lemmas, we assume that $X$ is a locally connected continuum with an endelement $E$. Define a relation, $\leqq$, in $X$ by $x \leqq y$ if, and only if, $x \in E$, or $x=y$, or $x$ separates $E$ and $y$ in $X$.

Lemma 12. The relation $\leqq$ is a semicontinuous quasi order. If $E$ is a single point, then $\leqq i$ a partial order.

Proof. That the relation is a quasi order, and is a partial order when $E$ is a point, is essentially proved in [14, Chap. III, 1.3]. To show that this quasi order is semicontinuous, it suffices to show $M(x)$ and $L(x)$ closed, for each $x \in X$. That $L(x)$ is closed follows from [14, Chap. III, 4.2]. If $x \in E$, then $M(x)=X$. If $x \in X-E$, then 


$$
M(x)=x \cup\{y: x \text { separates } E \text { and } y \text { in } X\} .
$$

If $C$ is the component of $X-x$ which contains $E$, then $M(x)=X-C$. Since $X$ is locally connected, $M(x)$ is closed.

Lemma 13. If $f(X)=X$ is non-alternating, and $f(E) \subset E$, then $f$ is order-preserving.

Proof. Let $x \leqq y$ in $X$. That $f(x) \leqq f(y)$ is trivial except in the case where $x$ separates $E$ and $y, f(x) \in X-E$, and $f(x) \neq f(y)$. Then $f^{-1} f(x)$ separates $E$ and $y$, so that by Lemma $11, f(x)$ separates $E$ and $f(y)$.

LEMma 14. If $f(X)=X$ is non-alternating, and $f(E) \subset E$, then there is a cutpoint $x$ of $X$ such that $x$ and $f(x)$ are comparable. Further, $x$ can be so chosen that, for some $y \in X, x<y$ and $x<f(y)$.

Proof. If $E$ contains a cutpoint $x$ of $X$, then $f(x) \in E$, so that $x$ and $f(x)$ are comparable. Since $f(X)=X$ and $f(E) \subset E$, there is $y \in X$ $-E$ such that $f(y) \in X-E$. Therefore, by Lemma $9, x<y$ and $x$ $<f(y)$. If $E$ contains no cutpoint of $X$, choose $y \in X$ such that $y \in X-E$ and $f(y) \in X-E$. Since $E$ is an endelement, there is an open set $A$ such that

$$
E \subset \bar{A} \subset X-(y \cup f(y))
$$

and $\bar{A}-A=x$, a cutpoint. Note that $x<y, x<f(y)$, and

$$
X-x=A \cup B, \quad A \mid B, \quad E \subset A, \quad y \cup f(y) \subset B .
$$

Note further that $f(y) \in B \cap f(B)$. If $f(x) \in \bar{B}$, then $x \leqq f(x)$, and if $f(x) \in E$, then $f(x) \leqq x$, so that in either case the lemma is proved. Otherwise, $f(x) \in A-E$; in this event we consider two cases.

Case 1. $f(x)$ is not a cutpoint. Then by Lemma $11, f^{-1} f(x)$ fails to cut $X$ and therefore $f^{-1} f(x)$ contains either $A$ or $B$. If $B \subset f^{-1} f(x)$, then $f(y) \in A$, a contradiction, and if $A \subset f^{-1} f(x)$, then $f(x) \in E$, likewise a contradiction.

Case 2. $f(x)$ is a cutpoint. In this case, we claim that

$$
\begin{gathered}
X-f(x)=(f(A)-f(x)) \cup(f(B)-f(x)), \\
f(A)-f(x) \neq 0 \neq f(B)-f(x), \quad f(A)-f(x) \mid f(B)-f(x) .
\end{gathered}
$$

Since $E \subset A$ and $f(x) \in X-E$, it follows that $f(E) \subset f(A)-f(x) \neq 0$. Since $f(x) \neq f(y) \in f(B)$, we have $f(B)-f(x) \neq 0$. To show

$$
f(A)-f(x) \mid f(B)-f(x),
$$

suppose there exists $t \in X$ such that

$$
t \in \overline{f(A)-f(x)} \cap(f(B)-f(x)) .
$$


Then $t \in f(\bar{A}) \cap f(B)$; since $f$ is non-alternating, $t=f(x)$, a contradiction. Similarly,

$$
(f(A)-f(x)) \cap \overline{f(B)-f(x)}=0,
$$

establishing the separation. If $x \in f(\bar{B})$, then $f(x) \leqq x$, proving the lemma. If $x \in f(A)-f(x)$, then $f(A)-f(x)$ meets both $A$ and $B$, and, since $f(x) \in A$ and $\bar{B}$ is connected, it follows that $\bar{B} \subset f(A)-f(x)$. But this implies that $f(y) \in f(A)-f(x)$, a contradiction.

Before proving our chief result, it is convenient to introduce a notion of clustering and convergence for sequences of sets in a topological space. (In connection with metric spaces, see [14, Chap. 1, 7].) A sequence of sets $A_{n}, n=1,2, \cdots$, is said to cluster at a point $x$ if infinitely many members of the sequence $A_{n}$ meet any given open neighborhood of $x$. The set of cluster points is denoted $\lim \sup A_{n}$. The sequence $A_{n}$ converges to $x$ if all but a finite number of the $A_{n}$ meet any given open neighborhood of $x$. The set of convergence points of $A_{n}$ is denoted $\lim \inf A_{n}$. Obviously, $\lim \inf A_{n} \subset \lim \sup A_{n}$. If $\lim \sup A_{n}=\lim \inf A_{n}$, then this set is denoted $\lim A_{n}$.

THEOREM 9. Let $X$ be a locally connected continuum with an endelement $E$, and $f(X)=X$ monotone such that $f(E)=E$. Then $X$ contains a non-null subcontinuum $K$ such that $f(K)=K$, and either $K$ is a cutpoint or $K \subset X-E$. Further, no point separates any pair of points of $K$ in $X$.

Proof. By Lemma $12, X$ is a QOTS, and by Lemma $13, f$ is orderpreserving. By Lemma 14, there is a cutpoint $x \in X$ such that $x$ and $f(x)$ are comparable. If $x \in E$, then by Lemma 9 ,

$$
X-x=(E-x) \cup(X-E), \quad E-x \mid X-E .
$$

If $f(x)=x$, the theorem is proved; otherwise $f(x) \in E-x$, and hence by Lemma $8, f(x)$ is not a cutpoint. Therefore, either $E \subset f^{-1} f(x)$ or $X-E \subset f^{-1} f(x)$. But $E \subset f^{-1} f(x)$ contradicts $f(E)=E$ and $X-E$ $C f^{-1} f(x)$ contradicts $f(X)=X$.

It remains to consider the case where $x \in X-E$. If $x \leqq f(x)$, then by Theorem 8 , there is a non-null set $K \subset E\left(x_{0}\right), x_{0} \in X-E$, such that $f(K)=K$. Since $E\left(x_{0}\right)=x_{0}$, the theorem is proved. If $f(x)<x$, let

$$
X-x=A \cup B, \quad A \mid B,
$$

where $A$ is the component of $X-x$ containing $E$; then $f(x) \in A$. Further, by Lemma 14, $x$ can be so chosen that there exists $b \in X$ such that $b \in B$ and $f(b) \in B$. Note that for any positive integer, $n$,

$$
X-f^{-n}(x)=f^{-n}(A) \cup f^{-n}(B), \quad f^{-n}(A) \mid f^{-n}(B) .
$$


Since $f$ is monotone, $f^{-1}(\bar{B})$ is connected, and, because $x \in f^{-1}(A)$, $b \in B$, and $f(b) \in B$, it follows that $f^{-1}(\bar{B}) \subset B$. Hence $\bar{A} \subset f^{-1}(A)$, and therefore for $n<m$, we have $f^{-n}(\bar{A})<f^{-m}(A)$. Since $X$ is compact, $\lim \sup f^{-n}(x) \neq 0$.

(A) $\lim \sup f^{-n}(x)=\lim \inf f^{-n}(x)$. For if not, there is $x_{0} \in \lim \sup f^{-n}(x)$ and a connected open set $U$ with $x_{0} \in U$, such that if $N$ is a positive integer with $f^{-N}(x) \cap U \neq 0$, then there is $m>N$ such that $f^{-m}(x) \cap U=0$. Either $U \subset f^{-m}(A)$ or $U \subset f^{-m}(B)$; since $f^{-N}(x) \cap U \neq 0$ and $f^{-N}(x) \subset f^{-m}(A)$, it follows that $U \subset f^{-m}(A)$. Therefore, for $p>m$, we have

$$
U \subset f^{-m}(A) \subset f^{-p}(A)
$$

so that $U \cap f^{-p}(x)=0$, contradicting our assumption that $x_{0}$ $\in \lim \sup f^{-n}(x)$.

(B) $\lim f^{-n}(x)$ is a continuum. It is trivial that $\lim f^{-n}(x)$ is closed and hence compact. Suppose

$$
\lim f^{-n}(x)=P \cup Q, \quad P \mid Q .
$$

Since $X$ is normal, there are disjoint open sets $U$ and $V$ such that $P \subset U, Q \subset V$. Assuming $P \neq 0 \neq Q$, we may choose a positive integer $N$ such that

$$
f^{-m}(x) \cap U \neq 0 \neq f^{-m}(x) \cap V
$$

for all $m \geqq N$. Since each $f^{-m}(x)$ is connected, we may choose a sequence $y_{n}, n=N, N+1, \cdots$, with each $y_{n} \in f^{-n}(x)-U \cup V$. Clearly $y_{n}$ clusters at some $y_{0} \in X-U \cup V$, contradicting $\lim f^{-n}(x) \subset U \cup V$. Therefore, $\lim f^{-n}(x)$ is connected and hence is a continuum.

(C) No point separates any pair of points of $\lim f^{-n}(x)$ in $X$. Otherwise, there is $a \in X, p$ and $q$ in $\lim f^{-n}(x)$ such that

$$
X-a=P \cup Q, \quad P \mid Q, \quad p \in P, \quad q \in Q .
$$

Since $f^{-n}(x)$ converges to both $p$ and $q$, there is a positive integer $N$ such that $f^{-m}(x)$ meets both $P$ and $Q$ for $m \geqq n$. By (B) we have

$$
a \in \cap\left\{f^{-m}(x): m \geqq N\right\},
$$

whence $f^{N+1}(a)=f(x)=x$, in contradiction of our assumption that $f(x)<x$.

It follows easily that $f\left(\lim f^{-n}(x)\right) \subset \lim f^{-n}(x)$, so that

$$
K=\bigcap\left\{f^{i}\left(\lim f^{-n}(x)\right): i=1,2, \cdots\right\}
$$

is a non-null continuum, $f(K)=K$, and no point separates any pair 
of points of $K$ in $X$. Since $\bar{A} \subset f^{-n}(A)$, for each $n=1,2, \cdots$, we conclude that

$$
K \subset X-\bar{A} \subset X-E .
$$

This completes the proof.

Corollary 9.1. If $E$ is a point, then $K \subset X-E$.

The theorem of Schweigert [9] and Wallace [10] follows easily from the machinery set up in this section.

Theorem 10 (SchWeigert-Wallace). Let $X$ be a locally connected continuum with an endelement $E$. If $f(X)=X$ is a homeomorphism such that $f(E)=E$, then $f$ has a fixed point $x_{0} \neq E$.

Proof. By Lemma 13, $f$ and $f^{-1}$ are order-preserving, and by Lemma 14 , there is a cutpoint $p$ of $X$ such that $p$ and $f(p)$ are comparable. If $p \in E$, then $f(p)$ is a cutpoint whence by Lemma $8, f(p)$ $=p$. If $p \in X-E$, either $p \leqq f(p)$ or $f(p) \leqq p$. If $p \leqq f(p)$, then by Theorem 8, there exists $K \subset E\left(x_{0}\right), x_{0} \in X-E$, such that $f(K)=K$. Since $E\left(x_{0}\right)=x_{0}$, the theorem is proved. If $f(p) \leqq p$, then by the same reasoning, $f^{-1}$ has a fixed point in $X-E$.

It is natural to inquire whether Theorem 10 holds if $f$ is nonalternating or monotone. We give a counterexample. Let $X$ consist of all points $(x, y, t)$ in 3 -space, with $0 \leqq t \leqq 2$, and satisfying

$$
\begin{array}{cc}
x^{2}+y^{2}=1-t^{2}, & \text { if } 0 \leqq t \leqq 1, \\
x=0=y, & \text { if } 1 \leqq t \leqq 2 .
\end{array}
$$

$X$ is a locally connected continuum, and $e=(0,0,2)$ is an endpoint. Define $f: X \rightarrow X$ by

$$
\begin{aligned}
f(x, y, t) & =\left(-\frac{(1-2 t) x}{\left(x^{2}+y^{2}\right)^{1 / 2}},-\frac{(1-2 t) y}{\left(x^{2}+y^{2}\right)^{1 / 2}}, 2 t\right) & \text { if } \quad 0 \leqq t \leqq 1 / 2, \\
f(x, y, t) & =(0,0,2 t) & \text { if } \quad 1 / 2 \leqq t \leqq 1, \\
f(x, y, t) & =e & \text { if } 1 \leqq t \leqq 2 .
\end{aligned}
$$

$f$ is continuous, monotone, and $f(e)=e$, but there are no other fixed points. Of course, the subcontinuum

$$
K=\{(x, y, t): t=0\}
$$

is fixed under $f$.

The techniques employed in proving Theorem 9 depend very strongly on local connectedness. Schweigert [9] has shown that if $X$ is a semi locally connected separable continuum with an endelement 
$E$, and $f(X)=X$ is a homeomorphism which keeps $E$ fixed, then there exists an $f$-invariant subcontinuum $K \neq E$, no pair of points of which can be separated by any point in $X$. Wallace [10] showed that semi local connectedness and separability may be dropped, and that in the presence of local connectedness, $K$ is a point (Theorem 10 above). The question we raise here is whether Theorem 9 is valid in the nonlocally connected case. While this conjecture seems reasonable, it is unlikely that the order-theoretic methods employed in this paper are adequate.

\section{BIBLIOGRAPHY}

1. G. Birkhoff, Lattice theory, rev. ed., New York, 1948.

2. S. Eilenberg, Ordered topological spaces, Amer. J. Math. vol. 63 (1941) pp. 3945. 582.

3. O. Frink, Topology in lattices, Trans. Amer. Math. Soc. vol. 51 (1942) pp. 569-

4. A. Haar and D. König, Über einfach geordnete Mengen, J. Reine Angew. Math. vol. 139 (1910) pp. 16-28.

5. J. L. Kelley, Convergence in topology, Duke Math. J. vol. 17 (1950) pp. 277-283.

6. L. Nachbin, Sur les espaces topologiques ordonnés, C. R. Acad. Sci. Paris vol. 226 (1948) pp. 381-382.

7. - Linear continuous functionals positive on the increasing continuous functions, Summa Brasiliensis Mathematicae vol. 2 (1951).

8. - Topologia e Ordem, Chicago, 1950.

9. G. E. Schweigert, Fixed elements and periodic types for homeomor phisms on s.l.c. continua, Amer. J. Math. vol. 66 (1944) pp. 229-244.

10. A. D. Wallace, $A$ fixed point theorem, Bull. Amer. Math. Soc. vol. 51 (1945) pp. $413-416$.

11. - Monotone transformations, Duke Math. J. vol. 9 (1943) pp. 487-506. 144.

12. ——, Endelements and the inversion of continua, ibid. vol. 16 (1949) pp. 141-

13. L. E. Ward, Jr., Binary relations in topological spaces, to appear.

14. G. T. Whyburn, Analytic topology, New York, 1942.

15. R. L. Wilder, Topology of manifolds, New York, 1949.

TULANE UNIVERSITY 